Check for updates

Cite this: Chem. Sci., 2019, 10, 5176

๑ All publication charges for this article have been paid for by the Royal Society of Chemistry

Received 4th February 2019

Accepted 16th April 2019

DOI: $10.1039 / \mathrm{c} 9 \mathrm{sc00633h}$

rsc.li/chemical-science

\title{
Brønsted acid and Pd-PHOX dual-catalysed enantioselective addition of activated C-pronucleophiles to internal dienes $\uparrow$
}

\author{
Sangjune Park, Nathan J. Adamson and Steven J. Malcolmson (DD*
}

We describe the development of $\mathrm{Pd}-\mathrm{PHOX}$-catalysed enantioselective couplings of internal dienes with malononitrile and other activated $\mathrm{C}$-pronucleophiles. Reactions are dramatically accelerated by the addition of $\mathrm{Et}_{3} \mathrm{~N} \cdot \mathrm{HBAr}_{4}{ }_{4}$ as a Brønsted acid co-catalyst, enabling a suite of products bearing a variety of alkyl substituents at the stereogenic carbon to be prepared. A series of mechanism-oriented experiments reveal key aspects of the catalytic cycle and the importance of the non-coordinating $\mathrm{BAr}_{4}{ }_{4}$ counterion in not only promoting reactions of internal dienes but also additions of previously unreactive nucleophiles towards terminal dienes.

\section{Introduction}

The addition of carbon-based electrophiles to enolates is a hallmark strategy in organic synthesis for the enantioselective formation of $\mathrm{C}-\mathrm{C}$ bonds. Mannich reactions, aldol and Michael additions $^{1}$ are among the most common approaches for the construction of small molecules. Enolate alkylations are also widespread but often focused towards controlling stereochemistry at a carbonyl's $\alpha$-position. ${ }^{2}$ An important class of enolate alkylation is allylic substitution, ${ }^{3}$ which enables the assembly of molecules comprised of carbonyls with $\beta$-stereogenic centres ${ }^{4}$ while often concomitantly setting the stereochemistry at the $\alpha$ position. ${ }^{5,6}$ Additionally, the products contain pendant unsaturation that might be leveraged for downstream synthesis applications. These enolate alkylations (allylic and non-allylic electrophiles) utilize preactivated electrophiles such as alkyl halides or allylic acetates.

The use of olefins as alkylating agents ${ }^{7}$ offers a number of distinct advantages, not the least of which is the inherent stability of an alkene compared to the lability of a leaving group, facilitating carrying the olefin electrophile through several transformations in a multistep sequence. A handful of researchers have explored this approach in enantioselective catalysis. For example, enol/enolate addition to allenes ${ }^{8}$ has afforded products with a variety of substituents at the $\beta$-stereogenic centre (Scheme 1), in some cases with additional control at the carbonyl's $\alpha$-position. ${ }^{8 a, c}$ Employing allenes as electrophiles has exclusively ${ }^{9}$ resulted in products bearing a terminal alkene. In a similar process, Rh-bis(phosphine)-

Department of Chemistry, Duke University, French Family Science Center, 124 Science Drive, Durham, NC, 27708, USA. E-mail: steven.malcolmson@duke.edu

$\dagger$ Electronic supplementary information (ESI) available. See DOI: $10.1039 / \mathrm{c} 9 \mathrm{sc} 00633 \mathrm{~h}$ catalysed enol additions to internal alkynes delivers analogous products. ${ }^{10} \neq$ Terminal dienes have also emerged as alkyl electrophiles capable of affording products comprised of internal alkenes. ${ }^{11}$ Recently, our group described the Pd-PHOXcatalysed addition of activated pro-enolates to these dienes, ${ }^{12-14}$ and Xiao, Zhou and co-workers demonstrated diene reactions in $\mathrm{Ni}$-bis(phosphine)-catalysed additions of enolates formed from simple ketones (Scheme 1). ${ }^{15}$ However, the products obtained from reactions of terminal dienes bear only a methyl group at the $\beta$-stereogenic centre.

In comparison, hydrofunctionalisation of internal dienes delivers molecules with both disubstituted olefins and myriad groups at the stereogenic $\beta$-position. Still, enantioselective additions to acyclic 1,4-disubstituted dienes are challenging: only indole ${ }^{16}$ and amine ${ }^{17}$ couplings have been reported. In this

Addition of Enol/Enolate Nucleophiles to Allenes and Terminal Dienes
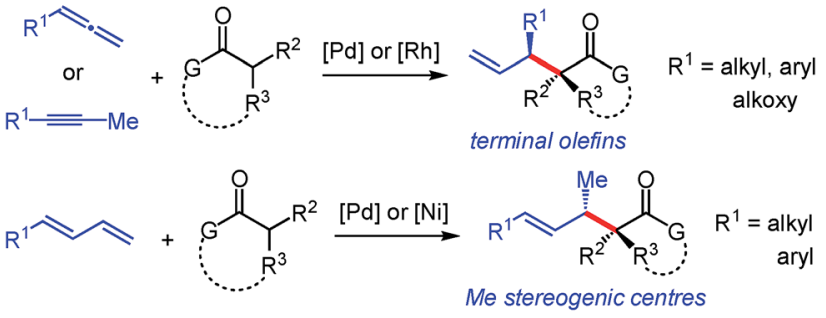

- This Work: Addition of Enolates to Internal Dienes
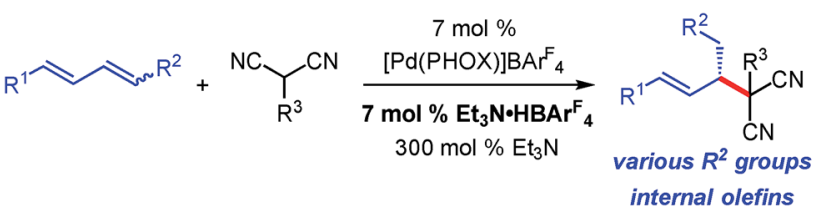

Scheme 1 Catalytic enantioselective additions of enols/enolates to olefins. 
work, we illustrate that malononitrile and related pronucleophiles undergo highly regio- and enantioselective additions to internal dienes (Scheme 1). High reaction efficiency was achievable by the combination of an electron-deficient PdPHOX catalyst and $\mathrm{Et}_{3} \mathrm{~N} \cdot \mathrm{HBAr}_{4}^{\mathrm{F}}$ as Brønsted acid co-catalyst.

\section{Results and discussion}

\section{Method development and product functionalisation}

We initiated our investigation by examining the addition of malononitrile to diene 1a with Pd-PHOX catalyst Pd-1, the optimal catalyst for internal diene hydroamination, ${ }^{17}$ which bears the non-coordinating $\mathrm{BAr}^{\mathrm{F}}{ }_{4}$ counterion (Table 1). We hypothesized that the small size of malononitrile, its low $\mathrm{p} K_{\mathrm{a}}{ }^{18}$ and the relatively high nucleophilicity of its anion ${ }^{19}$ would make it exceptionally reactive towards alkylation. Indeed, in the addition of malononitrile to 1-phenylbutadiene-a terminal diene-we found it difficult to suppress double alkylation. ${ }^{12}$ In the absence of any additive, there is no observable reaction with diene 1a (entry 1); however, with $300 \mathrm{~mol} \% \mathrm{Et}_{3} \mathrm{~N}$, the desired 2a is generated in $>99: 1$ er as the sole product of the reaction but in only $24 \%$ yield (entry 2 ).

We discovered that the addition of a substoichiometric quantity of $\mathrm{Et}_{3} \mathrm{~N} \cdot \mathrm{HBAr}_{4}^{\mathrm{F}}$ as a Brønsted acid greatly improves the product yield (entry 3 ). Notably, the addition of Brønsted acid has been observed as a crucial component in several other transformations involving nucleophilic additions to olefins, ${ }^{20}$

Table 1 Reaction optimization for diene-malononitrile coupling ${ }^{a}$

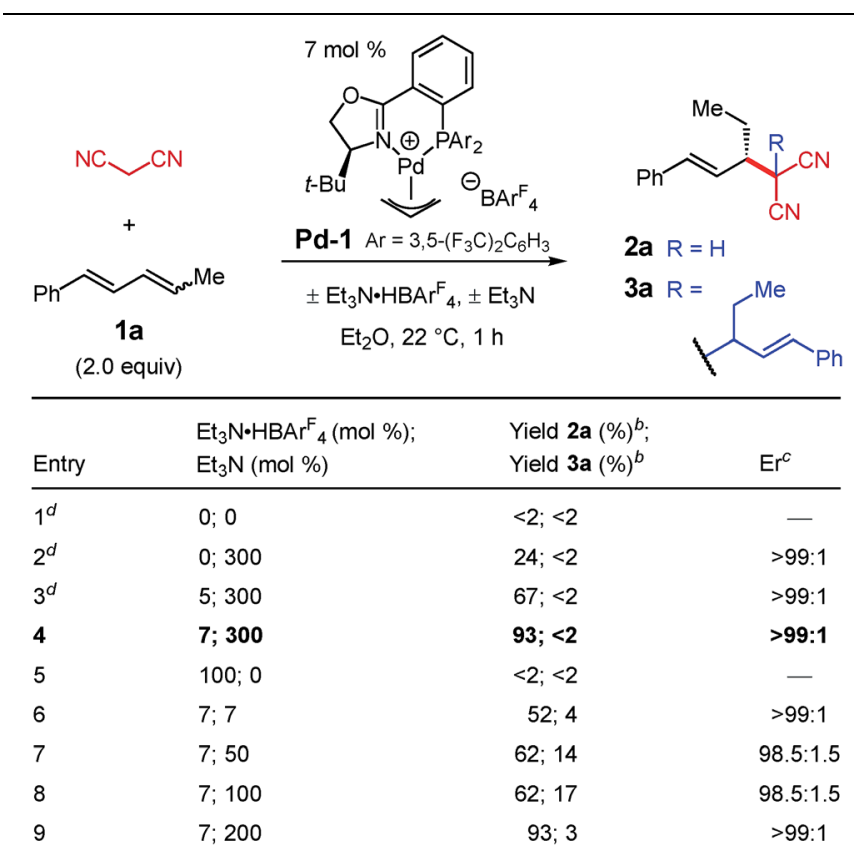

${ }^{a}$ Reaction under $\mathrm{N}_{2}$ with $0.2 \mathrm{mmol}$ malononitrile in $0.2 \mathrm{~mL} \mathrm{Et}_{2} \mathrm{O}$. 1a used as a 1.8:1 E,Z:E,E mixture of stereoisomers. See the ESI for experimental details. ${ }^{b}$ Isolated yield of purified product. ${ }^{c}$ Determined by HPLC analysis of purified 2. ${ }^{d}$ Reaction with $5 \mathrm{~mol} \%$ Pd-1. including reactions of enols. ${ }^{8 a-d, 10 a}$ Further raising the catalyst loading of both Pd-1 and $\mathrm{Et}_{3} \mathrm{~N} \cdot \mathrm{HBAr}_{4}^{\mathrm{F}}$ to $7 \mathrm{~mol} \%$, which proved to be the optimal conditions, allows $2 \mathrm{a}$ to be isolated in $93 \%$ yield and $>99: 1$ er (entry 4). In the absence of $\mathrm{Et}_{3} \mathrm{~N}$, even with stoichiometric $\mathrm{Et}_{3} \mathrm{~N} \cdot \mathrm{HBAr}^{\mathrm{F}}$ (entry 5), alkylation product $2 \mathrm{a}$ is not formed. Lesser quantities of the base, from $7 \mathrm{~mol} \%$ up to $100 \mathrm{~mol} \%$, drastically reduce the yield of $2 \mathrm{a}$ while also enabling its conversion to bis-alkylation product 3a (entries 6-8). With $200 \mathrm{~mol} \% \mathrm{Et}_{3} \mathrm{~N}$, product $2 \mathrm{a}$ is again isolated in $93 \%$ yield but is still accompanied by a small quantity of byproduct $\mathbf{3 a}$ (entry 9 ).

Under the optimal conditions, several 1,4-disubstituted dienes couple with malononitrile (Table 2). Electronic perturbations of the diene's aryl group are tolerated, affording 2b-d in $40-66 \%$ yield and $>95: 5$ er. Moving the aryl group substituent to the meta or ortho positions generates tolyl products 2e and 2f in higher yields (87-89\%) and with excellent enantioselectivity (97.5: 2.5 and $96: 4$ er, respectively). Changing the substituent at the 4-position of the diene enables products with a variety of alkyl groups at the stereogenic carbon to be obtained $(2 \mathbf{g}-\mathbf{k})$; the reaction is tolerant of both ethereal (2i-j) and carbonyl functionality (2h and $2 \mathbf{k})$. All

Table 2 Internal diene scope for malononitrile additions ${ }^{a}$

\begin{tabular}{|c|c|c|}
\hline $\begin{array}{l}\mathrm{NC} \rightleftharpoons \mathrm{CN} \\
+\end{array}$ & $\begin{array}{c}7 \mathrm{~mol} \% \mathrm{Pd}-1 \\
7{\mathrm{~mol} \% \mathrm{Et}_{3} \mathrm{~N} \cdot \mathrm{HBAr}_{4}}_{4} \\
300 \mathrm{~mol} \% \mathrm{Et}_{3} \mathrm{~N}\end{array}$ & \\
\hline $\begin{array}{c}\text { 1b-I } \\
\text { (2.0 equiv) }\end{array}$ & $\mathrm{Et}_{2} \mathrm{O}, 22^{\circ} \mathrm{C}, 1 \mathrm{~h}$ & $2 b-1$ \\
\hline
\end{tabular}

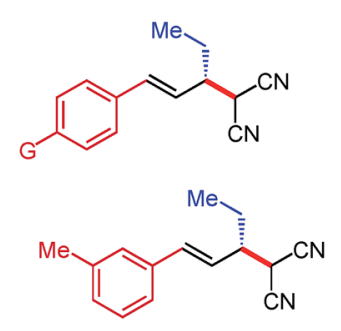

$2 \mathrm{e}$

$89 \%$ yield, $97.5: 2.5 \mathrm{er}$<smiles>N#CC[C](C=Cc1ccccc1)C(C#N)C#N</smiles>

$2 \mathrm{~g}$

$78 \%$ yield, 95.5:4.5 er<smiles>N#CC(C#N)[C@H](/C=C/c1ccccc1)O[AsH3]</smiles>

$2 \mathbf{j}^{b}$

$81 \%$ yield, $96: 4$ er

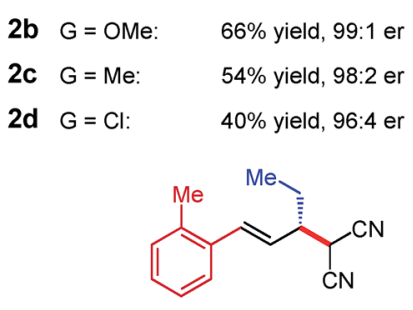

$2 f$

$87 \%$ yield, $96: 4$ er

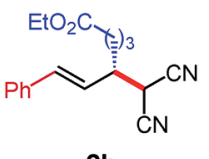

2h

$62 \%$ yield, $95.5: 4.5$ er<smiles>N#CC(C#N)C(/C=C/c1ccccc1)NNc1ccccc1</smiles>

$2 \mathbf{k}^{b}$

$44 \%$ yield, $90.5: 9.5 \mathrm{er}$

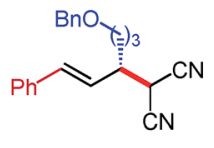

2i

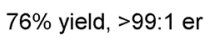<smiles>CCC(/C=C/Cl)C(C#N)C#N</smiles>

$2 \mathbf{I}^{c}$
$72 \%$ yield, $94: 6$ er

${ }^{a}$ Reaction under $\mathrm{N}_{2}$ with $0.2 \mathrm{mmol}$ malononitrile in $0.2 \mathrm{~mL} \mathrm{Et}_{2} \mathrm{O}$. Dienes were used as a mixture of stereoisomers $(1.1: 1$ to $4.2: 1$ $E, Z: E, E)$. See the ESI for experimental details. ${ }^{b}$ Isolated yield of purified product. ${ }^{c}$ Determined by HPLC analysis of purified 2 . 
aryl/alkyl-substituted diene substrates deliver malononitrile products $\mathbf{2 a - k}$ as a single regioisomer. Additionally, 1,4dialkyl-containing dienes also afford malononitrile addition products as a single regioisomer as long as the two alkyl groups are sterically differentiated. Vinylcyclohexane $2 \mathbf{l}$ is obtained in 72\% yield and 94:6 er. In all cases, enantioselectivity is constant throughout the course of the reaction, indicating that the process is irreversible.

Substituted malononitrile pronucleophiles undergo Pd-1catalysed addition to diene 1a within $1 \mathrm{~h}$ with complete regiocontrol (Table 3). The methyl-, benzyl-, and cinnamylsubstituted pronucleophiles generate products 5a-c in 78$99 \%$ yield and $>95: 5$ er. Acetylacetone, both less acidic ${ }^{21}$ and its anion less nucleophilic ${ }^{19}$ than malononitrile, leads to diketone 5d in 51\% yield and $92.5: 7.5$ er after $20 \mathrm{~h}$, still as a single regioisomer. Ethyl cyanoacetate addition to diene 1a, however, results in a $60: 40$ mixture of product regioisomers $5 \mathbf{e}$ and $6 \mathbf{e}(56 \%$ yield). Isomer $6 \mathbf{e}$ arises from addition of the nucleophile to the phenyl-substituted carbon of the $\pi$-allyl-Pd-1 intermediate derived from diene $\mathbf{1 a}$ whereas $5 \mathbf{e}$ results from nucleophile addition to the ethyl-substituted carbon of the same $\pi$-allyl complex. Dimethyl malonate, delivers a similar result (57: 43 5f : 6 f in $49 \%$ yield). Despite their lower $\mathrm{p} K_{\mathrm{a}}{ }^{\prime} \mathrm{s}^{21,22}$ but perhaps partially due to their lower nucleophilicities, ${ }^{19}$ cyclic pronucleophiles such as Meldrum's acid and dimedone

Table 3 Pronucleophile scope for internal diene addition reactions ${ }^{a, b, c}$
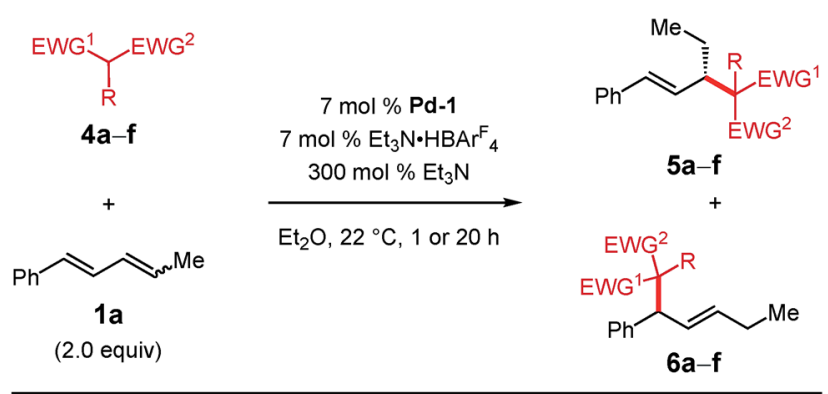

$1 \mathrm{~h}$ reactions:

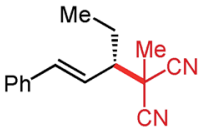

$5 a$

$99 \%$ yield, $96: 4$ er $>98: 2$ 5a:6a

$20 \mathrm{~h}$ reactions:

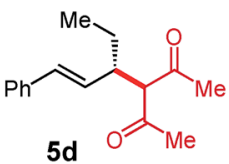

$51 \%$ yield, 92.5:7.5 er $>98: 2$ 5d:6d

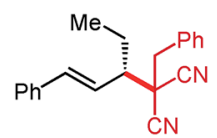

$5 b$

$90 \%$ yield, 95.5:4.5 er $>98: 2$ 5b:6b

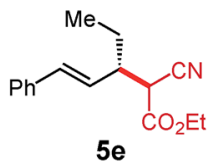

$56 \%$ yield $^{d}, 60: 40 \mathrm{dr}$ $60: 405 e: 6 e$

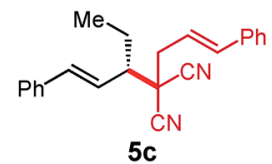

$78 \%$ yield, 95.5:4.5 er $>98: 25 c: 6 c$

${ }^{a}$ Reaction under $\mathrm{N}_{2}$ with $0.2 \mathrm{mmol}$ pronucleophile in $0.2 \mathrm{~mL} \mathrm{Et}_{2} \mathrm{O}$. 1a used as a 1.8:1 E,Z:E,E mixture of stereoisomers. ${ }^{b}$ Isolated yield of purified product. ${ }^{c}$ er determined by HPLC analysis of purified $\mathbf{5}$.

${ }^{d}$ Isolated yield of the isomeric mixture; er not determined.

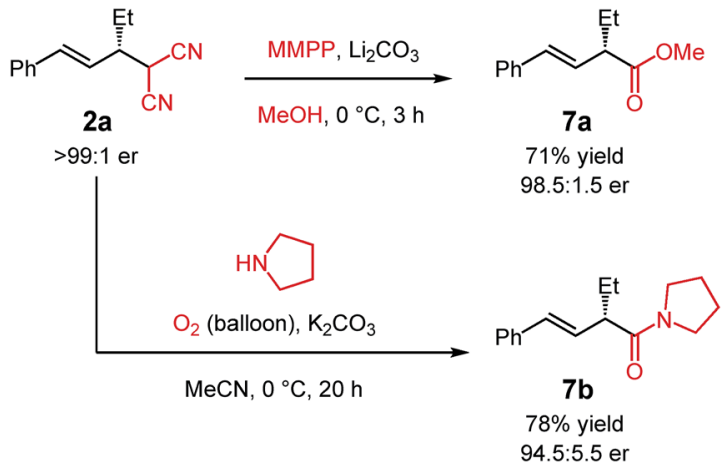

Scheme 2 Synthesis of $\alpha$-alkenyl carbonyls from the malononitrile group.

fail to undergo addition to diene 1a under the conditions shown in Table 3.

The malononitrile adducts of internal dienes are useful building blocks for further synthesis. For example, the malononitrile unit within 2a (Scheme 2) may undergo oxidative methanolysis ${ }^{23}$ via the cyanoketone to deliver $\alpha$-alkenylester $7 \mathbf{a}$ in $71 \%$ yield with minimal erosion of enantiopurity. Similarly, oxidation in the presence of pyrrolidine leads to $\alpha$-alkenylamide $7 \mathbf{b}$ in $78 \%$ yield $\left(94.5: 5.5\right.$ er). ${ }^{24}$

\section{Mechanism studies}

A mechanism for Pd-PHOX-catalysed addition of malononitrile to internal dienes is illustrated in Scheme 2.17,25 After initiation of Pd-1 by nucleophilic attack of the malononitrile anion upon its $\pi$-allyl ligand, coordination of diene 1 a to the $\operatorname{Pd}(0)$ species leads to intermediate i. Oxidative protonation at $\mathrm{Pd}$ by $\mathrm{Et}_{3} \mathrm{~N} \cdot \mathrm{HBAr}^{\mathrm{F}}{ }_{4}$ leads to hydrido complex ii, which undergoes migratory insertion, ultimately forming $\pi$-allyl-Pd species iii. The equilibration is accelerated and shifted towards complex iii by the addition of exogenous $\mathrm{Et}_{3} \mathrm{~N} \cdot \mathrm{HBAr}^{\mathrm{F}}{ }_{4}$ as the co-catalyst. At the same time, deprotonation of malononitrile by $\mathrm{Et}_{3} \mathrm{~N}$ leads to the malononitrile anion iv, an equilibrium that favours the neutral molecule. The anion may then attack $\pi$-allyl-Pd iii

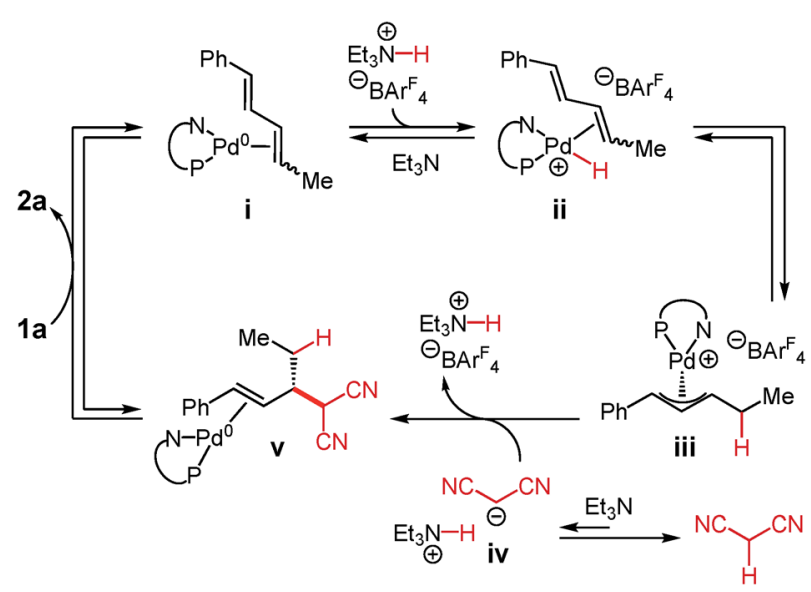

Scheme 3 Proposed mechanism of malononitrile addition to internal dienes. 
through an outer sphere pathway. The $\mathrm{C}-\mathrm{C}$ bond-forming event to form $\mathbf{v}$ appears to be irreversible and simultaneously regenerates $\mathrm{Et}_{3} \mathrm{~N} \cdot \mathrm{HBAr}^{\mathrm{F}}{ }_{4}$. Exchange of the olefin within $\mathbf{v}$ for diene 1a releases product $2 \mathbf{a}$ and regenerates complex $\mathbf{i}$.

In the context of this mechanistic proposal, we sought to address several facets of the Pd-PHOX-catalysed diene-enolate couplings. These investigations might allow us to glean further information about individual steps of this mechanism and more broadly understand diene couplings to nucleophiles promoted by Pd-PHOX catalysts.

We first set out to investigate the impact of diene stereochemistry upon the reaction. The transformations presented in Tables 1-3 utilize an $E, Z / E, E$-mixture of diene diastereomers favouring the $Z$-stereoisomer at the alkyl-substituted alkene. As shown in Scheme 4A, E,Z-1a is significantly more reactive than the $E, E$-stereoisomer, delivering malononitrile $2 \mathrm{a}$ in $90 \%$ yield and $99: 1$ er. Comparatively, E,E-1a (Scheme $4 \mathrm{~B}$ ) leads to only $35 \%$ yield of $2 \mathbf{a}(99: 1 \mathrm{er}$ ). In both experiments, one equivalent of diene was employed and any left unreacted was completely recovered. From the reaction of $E, Z-1 \mathbf{a}$, the recovered diene had almost completely isomerised to the $E, E$-diastereomer (Scheme $4 \mathrm{~A})$. In contrast, the recovered diene from reaction of $E, E-\mathbf{1 a}$ was still entirely that stereochemistry (Scheme 4B).

These data also help explain why under the optimal conditions, product 2a does not undergo a second alkylation event despite the presence of excess diene 1a (see Table 1). Of the two equivalents of diene 1a added to the reaction, only ca. 65\% (1.3 equiv.) is the more reactive $E, Z$-stereoisomer. The data also suggest the catalyst is capable of reacting with the diene and isomerising the $E, Z$ - to the $E, E$-diastereomer without product formation. Therefore, during the course of the reaction, as mono-alkylated product builds up, there is concomitant isomerisation of the diene occurring (likely along with catalyst decomposition), thereby slowing formation of bis-alkylated $\mathbf{3 a}$. With fewer $\mathrm{Et}_{3} \mathrm{~N}$ equivalents, $3 \mathbf{a}$ is observed to some degree (Table 1). This might be attributable to the equilibrium (see Scheme 3) between malononitrile and its deprotonated form iv being shifted towards the neutral molecule when less base is available, slowing the rate of formation of 2a. Mono-alkylated 2a that is formed may then engage in subsequent reaction with
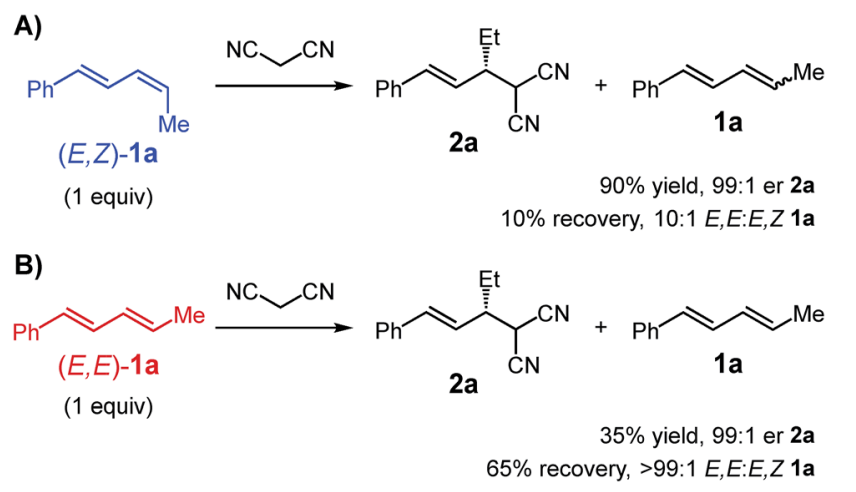

Scheme 4 Comparison of reactivity of diene diastereomers; conditions: $\mathrm{Pd}-1$ (7 mol\%), $\mathrm{Et}_{3} \mathrm{~N} \cdot \mathrm{HBAr}_{4}$ (7 mol\%), $\mathrm{Et}_{3} \mathrm{~N}$ (300 mol\%), $\mathrm{Et}_{2} \mathrm{O}$, $22{ }^{\circ} \mathrm{C}, 1 \mathrm{~h}$. remaining $E, Z$-1a prior to its consumption in product formation or its stereoisomerisation. Additionally, although substituted malononitriles, such as those shown in Table 3, are competent reaction partners, compound $\mathbf{2 a}$ is significantly more hindered ( $\beta$-branching) which slows a second alkylation event.

We next further sought to assess the reversibility of the individual elementary steps connecting diene 1a to $\pi$-allyl-Pd iii (Scheme 3). To do so, we examined the coupling of a deuterated pronucleophile, (1) to discover the label's position in the anticipated addition product with respect to the site of $\mathrm{C}-\mathrm{C}$ bond formation and (2) to determine whether deuterium could be incorporated into the diene without product forming. We chose deuterated 2-methylmalononitrile $(\boldsymbol{d}$-4a) as the pronucleophile (Scheme 5). Incredibly, although the protic version of 4a delivers $5 \mathrm{a}$ in $99 \%$ yield after $1 \mathrm{~h}$, no coupled product is formed after $20 \mathrm{~h}$ with $\boldsymbol{d}$-4a. This is likely due to the combination of a significant kinetic isotope effect and catalyst decomposition. Diene 1a could be completely recovered from the mixture: a portion of the diene had isomerised from the $E, Z$ isomer to $(E, E)$-1a (from $1.8: 1$ to $1: 1.7 E, Z: E, E)$. A significant percentage of the deuterium label ( $c a .43 \%)$ was transferred from $\boldsymbol{d}$-4a to the diene with the label confined solely to the recovered $E, E-1 \mathrm{a}$ and roughly evenly distributed between $\mathrm{C} 1$ and $\mathrm{C} 4$.

Just as in hydroamination of 1a with Pd-1 (reaction of Ndeuterated indoline), ${ }^{17}$ the kinetic isotope effect observed here is likely at least partially due to an equilibrium isotope effect that exchanges deuterium from $\boldsymbol{d}$-4a to diene $\mathbf{1 a}$ as mediated by $\mathrm{Et}_{3} \mathrm{~N}$ and the Pd catalyst. The lack of label in recovered $(E, Z)-1 \mathbf{a}$ is owed to its conversion exclusively to $\boldsymbol{d}-(E, E)-1 \mathrm{a}$ when it reacts with Pd-D: stereochemical isomerisation of the alkene occurs by Pd-D migratory insertion to $(E, Z)-\mathbf{1 a}$, bond rotation, and preferential $\beta$-hydride elimination (compared to $\beta$-deuteride elimination). As mentioned with regard to the reactivity difference of the two diene stereoisomers (vide supra), this process does not occur to incorporate deuterium into $(E, Z)-\mathbf{1 a}$ via reaction of $(E, E)$-1a. Finally, the distribution of the label in $\boldsymbol{d}-(E, E)-\mathbf{1 a}$ indicates that palladium-hydride/deuteride insertion may occur at either olefin of the diene, yet only insertion at the alkylsubstituted olefin leads to product in the reactions presented in Tables 1-3. The results further confirm that $E, Z$-diene coordination to Pd is reversible.

It is noteworthy that whereas malononitrile and acetylacetone nucleophiles afford a single detectable product

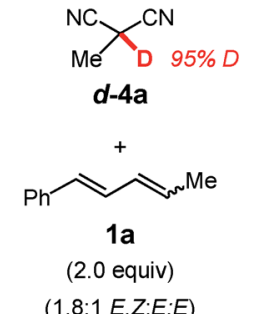

Scheme 5 Deuterium methylmalononitrile
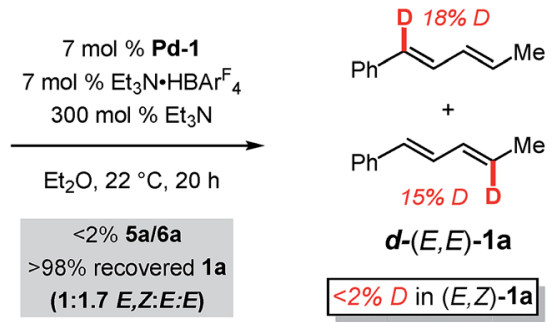

$<2 \% D$ in $(E, Z)-1 \mathrm{a}$ labelling studies

with deuterated 
regioisomer (Tables 1-3), ethyl cyanoacetate and dimethylmalonate deliver a mixture (5e/6e and $\mathbf{5 f} / \mathbf{6 f}$, respectively, Table 3). As mentioned earlier, the product isomers arise from attack of the nucleophiles upon two different carbons of the same $\pi$-allyl. Differing regioselectivities among nucleophiles is likely due to Curtin-Hammett kinetics in the reaction (Scheme 6) and is the combination of three properties of the nucleophile: (1) the $\mathrm{p} K_{\mathrm{a}}$ of the pronucleophile (affecting its equilibrium with the active deprotonated nucleophile), (2) the nucleophilicity of the carbanion, ${ }^{19}$ and (3) the sterics of the nucleophile. As documented previously for Pd-PHOX complexes, ${ }^{3 a}$ the $\pi$-allyl-Pd species exist as a mixture of the thermodynamically preferred exo-diastereomer and its isomeric endo-complex (Scheme 6). In this transformation, faster attack of the nucleophile upon the exoisomer at the carbon trans to the phosphine (ethyl-substituted carbon) leads to the observed major enantiomer and regioisomer ( 2 or 5 ). Conversely, some nucleophiles undergo competitive addition to the endo-diastereomer, attacking the phenyl-substituted carbon (trans to the phosphine), affording 6 . Although ethyl cyanoacetate has a similar $\mathrm{p} K_{\mathrm{a}}$ (ref. 26) to acetylacetone, generating a certain quantity of active nucleophile, its nucleophilicity is considerably greater (similar to malononitrile $\left.{ }^{19}\right)$. Likewise, even though dimethyl malonate has an even higher $\mathrm{p} K_{\mathrm{a}}{ }^{22}$ its nucleophilicity is greater still. ${ }^{19}$ Therefore, it is possible that reactants with greater nucleophilicity lead to similar rates of attack upon the exo- and endo-diastereomers of the $\pi$-allyl intermediate. The outlier is malononitrile, whose lower $\mathrm{p} K_{\mathrm{a}}$ (ref. 18) and high nucleophilicity ${ }^{19}$ should seemingly lead to an isomeric mixture. The high selectivity for products 2 or 5 with malononitrile (via the exo-isomer) is likely due to the small size of this pronucleophile.

An interesting dichotomy between reactions of terminal dienes, such as 1-phenylbutadiene, and those of internal dienes with activated C-pronucleophiles as catalysed by PdPHOX complexes presented itself in this study. In our previous investigations of $\mathrm{C}$-pronucleophile additions to
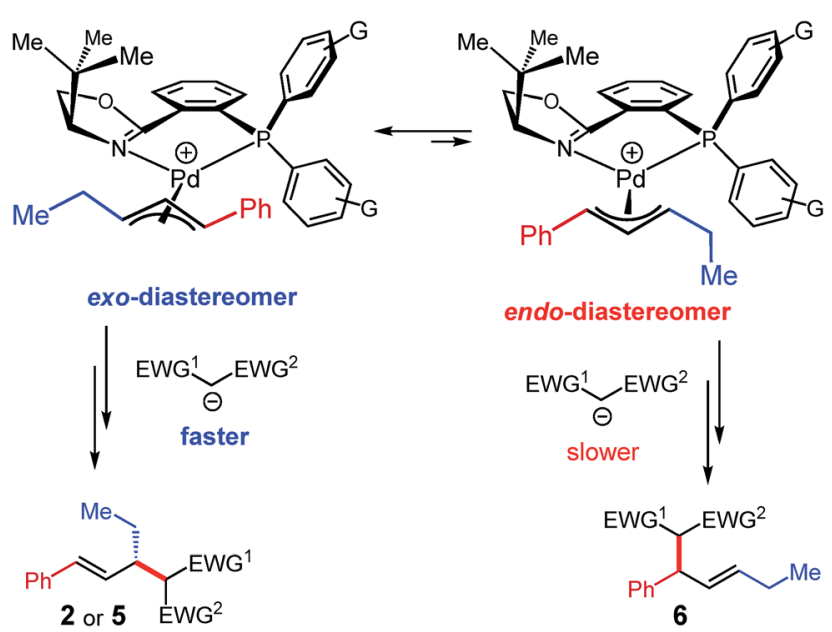

Scheme 6 Curtin-Hammett kinetics likely operative in internal diene-enolate couplings and responsible for the observed regio- and enantioselectivity. terminal dienes, ${ }^{\mathbf{1 2}}$ Meldrum's acid proved to be one of the best and most versatile pronucleophiles. Those reactions are promoted by the same PHOX ligand for Pd as in Pd-1 but with a catalyst that bears a tetrafluoroborate counterion in place of $\mathrm{BAr}^{\mathrm{F}}{ }_{4}$. Additionally, terminal diene reactions do not require Brønsted acid additive. Contrastingly, dimethyl malonate fails to undergo addition to 1-phenylbutadiene under the previously established optimal terminal diene conditions with the $\mathrm{BF}_{4}$-containing catalyst.

We wished to determine whether these differences arise from the $\mathrm{BAr}_{4}^{\mathrm{F}}$ counterion of Pd-1 or the $\mathrm{Et}_{3} \mathrm{~N} \cdot \mathrm{HBAr}^{\mathrm{F}}{ }_{4}$ additive. As shown in Scheme 7, dimethyl malonate $4 \mathbf{f}$ is able to add to 1-phenylbutadiene in the presence of $\mathbf{P d - 1}\left(\mathrm{BAr}_{4}^{\mathrm{F}}\right.$ counterion). Although the yield is slightly higher with the addition of $\mathrm{Et}_{3} \mathrm{~N} \cdot \mathrm{HBAr}^{\mathrm{F}}{ }_{4}$, both the regioselectivity and enantiopurity of the major product (8) are the same with or without Brønsted acid. Therefore, higher $\mathrm{p} K_{\mathrm{a}}$ pronucleophiles may react with terminal dienes with a Pd-PHOX catalyst comprised of the non-coordinating $\mathrm{BAr}^{\mathrm{F}}$. It is noteworthy that the regiomeric ratio favouring the 4,3-addition product 9 is higher for dimethyl malonate coupling to phenylbutadiene than to internal diene 1a ( $c f$., Table 3, compound $\mathbf{5 f})$.

We finally turned to addressing the lack of reactivity of Meldrum's acid towards internal diene 1a with Pd-1. In fact, not only does $\mathrm{C}-\mathrm{C}$ bond formation fail to occur in this case, based on the diene recovered from the reaction mixture, the initial $1.8: 1 E, Z: E, E$ ratio of 1 a also remains unchanged. The absence of stereochemical isomerisation indicates that catalyst formed from Pd-1 fails to engage the diene at all in the presence of this pronucleophile.

As mentioned, we previously developed the addition of Meldrum's acid to 1-phenylbutadiene with $\left[\left(\eta^{3}-\mathrm{C}_{3} \mathrm{H}_{5}\right)\right.$ $\mathrm{Pd}(\mathrm{PHOX})] \mathrm{BF}_{4}$ as the catalyst. ${ }^{12}$ Unfortunately, reactions of internal dienes are completely inhibited by having a $\mathrm{BF}_{4}$ counterion in the reaction medium, preventing our examining Meldrum's acid reaction with internal diene 1a catalysed by $\left[\left(\eta^{3}-\right.\right.$ $\left.\left.\mathrm{C}_{3} \mathrm{H}_{5}\right) \mathrm{Pd}(\mathrm{PHOX})\right] \mathrm{BF}_{4}$. However, Meldrum's acid addition to 1phenylbutadiene occurs smoothly with $\mathrm{BAr}^{\mathrm{F}}{ }_{4}$-containing Pd-1, delivering a nearly identical result to its $\mathrm{BF}_{4}$ analogue (Scheme 8). These data highlight that the acidic Meldrum's acid is compatible with Pd-1 no matter what its counteranion as long as a more reactive terminal diene is present. This suggests that

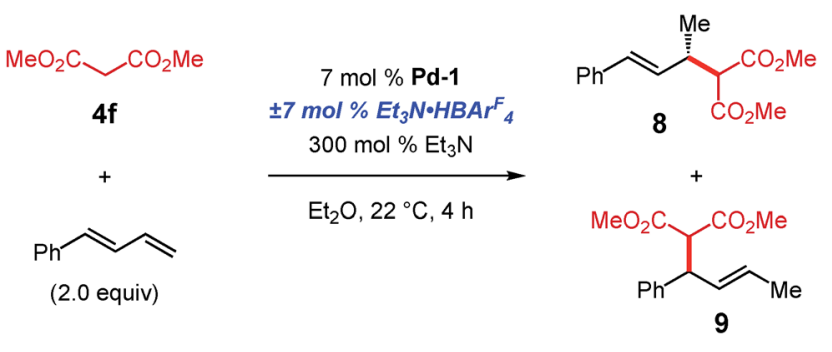

with $\mathrm{Et}_{3} \mathrm{~N} \cdot \mathrm{HBAr}_{4}: 76 \%$ yield, 88.5:11.5 er, 83:17 8:9 without $\mathrm{Et}_{3} \mathrm{~N} \cdot \mathrm{HBAr}_{4}: 59 \%$ yield, 88.5:11.5 er, 83:17 8:9

Scheme 7 Addition of dimethyl malonate to phenylbutadiene with Pd-1. 


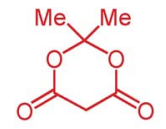

(1.5 equiv)

$+$

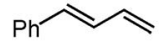

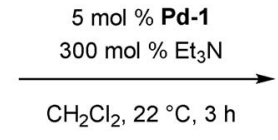

with $\left[\left(\eta^{3}-\mathrm{C}_{3} \mathrm{H}_{5}\right) \mathrm{Pd}(\mathrm{PHOX})\right] \mathrm{BAr}_{4}$ : $88 \%$ yield, $96.5: 3.5$ er with $\left[\left(\eta^{3}-\mathrm{C}_{3} \mathrm{H}_{5}\right) \mathrm{Pd}(\mathrm{PHOX}) \mathrm{BF}_{4}\right.$ : (ref. 12) $81 \%$ yield, $97.5: 2.5 \mathrm{er}$
Scheme 8 Meldrum's acid addition to phenylbutadiene with $\mathrm{Pd}$ $\mathrm{PHOX}$ catalysts bearing different counteranions.

as the internal diene $\mathbf{1 a}$ is more difficult to coordinate to the metal centre, significant catalyst decomposition occurs under the more acidic conditions imposed with Meldrum's acid (lower $\mathrm{p} K_{\mathrm{a}}$ ) compared to other pronucleophiles, thereby impeding the desired reaction pathway.

\section{Conclusions}

We have demonstrated that internal dienes act effectively as alkylating agents for enantioselective intermolecular couplings with in situ-generated enolates under Pd-PHOX catalysis. The atom economic olefin hydrofunctionalisations are accelerated by the addition of $\mathrm{Et}_{3} \mathrm{~N} \cdot \mathrm{HBAr}^{\mathrm{F}}{ }_{4}$ as a Brønsted acid co-catalyst. Somewhat counterintuitively, the use of superstoichiometric $\mathrm{Et}_{3} \mathrm{~N}$ is needed to generate sufficient quantities of the active nucleophile in order to form the mono-alkylation product selectively (i.e., avoid over-alkylation). Several features of the reaction mechanism have been uncovered through deuterium labelling the pronucleophile and by selectively employing different diastereomerically pure diene stereoisomers in reactions. Notable differences in participating C-pronucleophiles in Pd-PHOX-catalysed reactions of internal versus terminal 1,3dienes have been unveiled. Further investigation of reaction mechanism and applications to additional methodology development from lessons learned in these studies are ongoing.

\section{Conflicts of interest}

There are no conflicts to declare.

\section{Acknowledgements}

This work was supported by the National Science Foundation (CHE-1800012), the ACS Petroleum Research Fund (56575DNI1), and Duke University. N. J. A. is grateful to the Duke Chemistry Department for a Burroughs-Wellcome Fellowship.

\section{Notes and references}

\$ Reactions of alkynes proceed through the identical metal- $\pi$-allyl intermediate as those of allenes.

1 For reviews, see: (a) J. S. Johnson and D. A. Evans, Acc. Chem. Res., 2000, 33, 324; (b) S. Mukherjee, J. W. Yang, S. Hoffmann and B. List, Chem. Rev., 2007, 107, 5471; (c) S. Kobayashi,
Y. Mori, J. S. Fossey and M. M. Salter, Chem. Rev., 2011, 111, 2626; (d) G. L. Beutner and S. E. Denmark, Angew. Chem., Int. Ed., 2013, 52, 9086; (e) H. Pellissier, Chem. Rev., 2016, 116, 14868.

2 (a) T. Ooi, T. Miki, M. Shiraishi, M. Takeuchi and K. Maruoka, Angew. Chem., Int. Ed., 2003, 42, 3796; (b) A. G. Doyle and E. N. Jacobsen, J. Am. Chem. Soc., 2005, 127, 62; (c) H.-Y. Jang, J.-B. Hong and D. W. C. MacMillan, J. Am. Chem. Soc., 2007, 129, 7004; (d) S. Hong, J. Lee, M. Kim, Y. Park, C. Park, M.-h. Kim, S.-s. Jew and H.-g. Park, J. Am. Chem. Soc., 2011, 133, 4924; (e) T. Kano, Y. Hayashi and K. Maruoka, J. Am. Chem. Soc., 2013, 135, 7134; $(f)$ B. Teng, W. Chen, S. Dong, C. W. Kee, D. A. Gandamana, L. Zong and C.-H. Tan, J. Am. Chem. Soc., 2016, 138, 9935.

3 For reviews, see: (a) G. Helmchen and A. Pfaltz, Acc. Chem. Res., 2000, 33, 336; (b) B. M. Trost and M. L. Crawley, Chem. Rev., 2003, 103, 2921; (c) J. T. Mohr and B. M. Stoltz, Chem.Asian J., 2007, 2, 1476; (d) Z. Lu and S. Ma, Angew. Chem., Int. Ed., 2008, 47, 258; (e) J. C. Hethcox, S. E. Shockley and B. M. Soltz, ACS Catal., 2016, 6, 6207.

4 (a) B. M. Trost and C. B. Lee, J. Am. Chem. Soc., 2001, 123, 3671; (b) G. Lipowsky, N. Miller and G. Helmchen, Angew. Chem., Int. Ed., 2004, 43, 4595; (c) D. J. Weix and J. F. Hartwig, J. Am. Chem. Soc., 2007, 129, 7720; (d) B. Mao, Y. Ji, M. Fañanás-Mastral, G. Caroli, A. Meetsma and B. L. Feringa, Angew. Chem., Int. Ed., 2012, 51, 3168; (e) W. Chen and J. F. Hartwig, J. Am. Chem. Soc., 2012, 134, 15249; ( $f$ ) M. Chen and J. F. Hartwig, Angew. Chem., Int. Ed., 2014, 53, 8691; $(g)$ J. C. Hethcox, S. E. Shockley and B. M. Stoltz, Angew. Chem., Int. Ed., 2018, 57, 1; (h) S.-B. Tang, X. Zhang, H.-F. Tu and S.-L. You, J. Am. Chem. Soc., 2018, 140, 7737; (i) T. W. Butcher and J. F. Hartwig, Angew. Chem., Int. Ed., 2018, 57, 13125.

5 (a) B. M. Trost and C. Lee, J. Am. Chem. Soc., 2001, 123, 12191; (b) W.-H. Zheng, B.-H. Zheng, Y. Zhang and X.-L. Hou, J. Am. Chem. Soc., 2007, 129, 7718; (c) W. Chen and J. F. Hartwig, J. Am. Chem. Soc., 2013, 135, 2068; (d) S. Krautwald, D. Sarlah, M. A. Schafroth and E. M. Carreira, Science, 2013, 340, 1065; (e) W.-B. Liu, C. M. Reeves and B. M. Stoltz, J. Am. Chem. Soc., 2013, 135, 17298; ( $f$ ) W. Chen, M. Chen and J. F. Hartwig, J. Am. Chem. Soc., 2014, 136, 15825; (g) K. Ikeda, T. Futamura, T. Hanakawa, M. Minakawa and M. Kawatsura, Org. Biomol. Chem., 2016, 14, 3501; (h) X. Jiang, W. Chen and J. F. Hartwig, Angew. Chem., Int. Ed., 2016, 55, 5819; (i) K. Balaraman and C. Wolf, Angew. Chem., Int. Ed., 2017, 56, 1390; (j) J. Liu, Z. Han, X. Wang, F. Meng, Z. Wang and K. Ding, Angew. Chem., Int. Ed., 2017, 56, 5050; (k) P. Starkov, J. T. Moore, D. C. Duquette, B. M. Stoltz and I. Marek, J. Am. Chem. Soc., 2017, 139, 9615.

6 For examples where only an $\alpha$-stereogenic centre is set, see: (a) B. M. Trost and G. M. Schroeder, J. Am. Chem. Soc., 1999, 121, 6759; (b) J. T. Mohr, T. Nishimata, D. C. Behenna and B. M. Stoltz, J. Am. Chem. Soc., 2006, 128, 11348; (c) B. M. Trost, R. Koller and B. Schäffner, Angew. Chem., Int. Ed., 2012, 51, 8290; (d) T. B. Wright and 
P. A. Evans, J. Am. Chem. Soc., 2016, 138, 15303; (e) A. Ngamnithiporn, C. Jette, S. Bachman, S. C. Virgil and B. M. Stoltz, Chem. Sci., 2018, 9, 2547.

7 For reviews, see: (a) F. Dénès, A. Pérez-Luna and F. Chemla, Chem. Rev., 2010, 110, 2366; (b) Z. Dong, Z. Ren, S. J. Thompson, Y. Xu and G. Dong, Chem. Rev., 2017, 117, 9333.

8 (a) B. M. Trost, C. Jäkel and B. Plietker, J. Am. Chem. Soc., 2003, 125, 4438; (b) B. M. Trost, A. B. C. Simas, B. Plietker, C. Jäkel and J. Xie, Chem.-Eur. J., 2005, 11, 7075; (c) B. M. Trost, J. Xie and J. D. Sieber, J. Am. Chem. Soc., 2011, 133, 20611; (d) T. M. Beck and B. Breit, Angew. Chem., Int. $E d ., 2017,56,1903$. For an example that leads to products with only an $\alpha$-stereogenic centre, see: ; $(e) \mathrm{H}$. Zhou, Y. Wang, L. Zhang, M. Cai and S. Luo, J. Am. Chem. Soc., 2017, 139, 3631.

9 For a non-enantioselective exception, see: B. M. Trost and V. J. Gerusz, J. Am. Chem. Soc., 1995, 117, 5156.

10 For an enantioselective intermolecular example, see: $(a)$ F. A. Cruz and V. M. Dong, J. Am. Chem. Soc., 2017, 139, 1029For non-enantioselective intermolecular examples, see: (b) F. A. Cruz, Z. Chen, S. I. Kurtoic and V. M. Dong, Chem. Commun., 2016, 52, 5836; (c) C. Li, C. P. Grugel and B. Breit, Chem. Commun., 2016, 52, 5840.

11 For reviews regarding enantioselective reactions of dienes, see: (a) Y. Xiong, Y. Sun and G. Zhang, Tetrahedron Lett., 2018, 59, 347; (b) M. Holmes, L. A. Schwartz and M. J. Krische, Chem. Rev., 2018, 118, 6026.

12 N. J. Adamson, K. C. E. Wilbur and S. J. Malcolmson, J. Am. Chem. Soc., 2018, 140, 2761.

13 For an example that leads to products with only an $\alpha$ stereogenic centre, see: Y.-L. Su, L.-L. Li, X.-L. Zhou, Z.-Y. Dai, P.-S. Wang and L.-Z. Gong, Org. Lett., 2018, 20, 2403.

14 For intermolecular non-enantioselective enol/enolate additions to dienes, see: (a) K. Takahashi, A. Miyake and G. Hata, Bull. Chem. Soc. Jpn., 1972, 45, 1183; (b) B. M. Trost and L. Zhi, Tetrahedron Lett., 1992, 33, 1831; (c) A. Leitner, J. Larsen, C. Steffens and J. F. Hartwig, J. Org.
Chem., 2004, 69, 7552; (d) M. J. Goldfogel and S. J. Meek, Chem. Sci., 2016, 7, 4079; (e) M. J. Goldfogel, C. C. Roberts, R. S. Manan and S. J. Meek, Org. Lett., 2017, 19, 90.

15 L. Cheng, M.-M. Li, L.-J. Xiao, J.-H. Xie and Q.-L. Zhou, J. Am. Chem. Soc., 2018, 140, 11627.

16 J. S. Marcum, C. C. Roberts, R. S. Manan, T. N. Cervarich and S. J. Meek, J. Am. Chem. Soc., 2017, 139, 15580.

17 S. Park and S. J. Malcolmson, ACS Catal., 2018, 8, 8468.

18 W. S. Matthews, J. E. Bares, J. E. Bartmess, F. G. Bordwell, F. J. Cornforth, G. E. Drucker, Z. Margolin, R. J. McCallum, G. J. McCollum and N. R. Vanier, J. Am. Chem. Soc., 1975, 97, 7006.

19 R. Lucius, R. Loos and H. Mayr, Angew. Chem., Int. Ed., 2002, 41, 91.

20 (a) M. Kawatsura and J. F. Hartwig, J. Am. Chem. Soc., 2000, 122, 9546; (b) O. Löber, M. Kawatsura and J. F. Hartwig, J. Am. Chem. Soc., 2001, 123, 4366; (c) J. Pawlas, Y. Nakao, M. Kawatsura and J. F. Hartwig, J. Am. Chem. Soc., 2002, 124, 3669; (d) M. Utsunomiya and J. F. Hartwig, J. Am. Chem. Soc., 2004, 126, 2702; (e) A. B. Pritzius and B. Breit, Angew. Chem., Int. Ed., 2015, 54, 15818; (f) К. Xu, Y.-H. Wang, V. Khakyzadeh and B. Breit, Chem. Sci., 2016, 7, 3313; (g) X.-H. Yang and V. M. Dong, J. Am. Chem. Soc., 2017, 139, 1774; (h) X.-H. Yang, A. Lu and V. M. Dong, J. Am. Chem. Soc., 2017, 139, 14049; (i) C. P. Grugel and B. Breit, Org. Lett., 2018, 20, 1066; (j) S.-Z. Nie, R. T. Davison and V. M. Dong, J. Am. Chem. Soc., 2018, 140, 16450.

21 W. N. Olmstead and F. G. Bordwell, J. Org. Chem., 1980, 45, 3299.

22 E. M. Arnett, S. G. Maroldo, S. L. Schilling and J. A. Harrelson, J. Am. Chem. Soc., 1984, 106, 6759.

23 S. Förster, O. Tverskoy and G. Helmchen, Synlett, 2008, 2803. 24 B. H. Oh, I. Nakamura and Y. Yamamoto, Angew. Chem., Int. Ed., 2016, 55, 9060.

25 A. M. Johns, M. Utsunomiya, C. D. Incarvito and J. F. Hartwig, J. Am. Chem. Soc., 2006, 128, 1828-1839.

26 F. G. Bordwell and H. E. Fried, J. Org. Chem., 1981, 46, 4327. 